\title{
Selective Processing of Vestibular Reafference during Self-Generated Head Motion
}

\author{
Jefferson E. Roy and Kathleen E. Cullen \\ Aerospace Medical Research Unit, McGill University, Montreal, Quebec, Canada H3G 1 Y6
}

The vestibular sensory apparatus and associated vestibular nuclei are generally thought to encode head-in-space motion. Angular head-in-space velocity is detected by vestibular hair cells that are located within the semicircular canals of the inner ear. In turn, the afferent fibers of the vestibular nerve project to neurons in the vestibular nuclei, which, in head-restrained animals, similarly encode head-in-space velocity during passive whole-body rotation. However, during the active head-on-body movements made to generate orienting gaze shifts, neurons in the vestibular nuclei do not reliably encode head-in-space motion. The mechanism that underlies this differential processing of vestibular information is not known. To address this issue, we studied vestibular nuclei neural responses during passive head rotations and during a variety of tasks in which alert rhesus monkeys voluntarily moved their heads relative to space. Neurons similarly encoded head-in-space velocity during passive rotations of the head relative to the body and during passive rotations of the head and body together in space. During all movements that were generated by activation of the neck musculature (voluntary head-on-body movements), neurons were poorly modulated. In contrast, during a task in which each monkey actively "drove" its head and body together in space by rotating a steering wheel with its arm, neurons reliably encoded head-in-space motion. Our results suggest that, during active head-on-body motion, an efferent copy of the neck motor command, rather than the monkey's knowledge of its selfgenerated head-in-space motion or neck proprioceptive information, gates the differential processing of vestibular information at the level of the vestibular nuclei.

Key words: vestibular nucleus; self-motion; reafference; efference copy; gaze shift; gaze pursuit; vestibular reflexes; head-unrestrained
On the basis of behavioral experiments, von Holst and Mittelstaedt (1950) proposed that the sensory signals that arise from an animal's own movement, which they termed reafference, could be distinguished from sensory signals generated by external sources. They postulated that a copy of the motor command (efference copy) is combined with the afferent sensory signal to selectively remove the reafferent component caused by the motor behavior. Indeed, it is now well established that, in certain model systems, for example the electrosensory system of the electric fish (Bell, 1981) and mechanosensory system of the crayfish (Krasne and Bryan, 1973; Edwards et al., 1999), sensory signals are suppressed at the level of afferent fibers and/or the central neurons to which they project, via an efference copy signal. Accordingly, sensory information is filtered such that signals arising from external sources are emphasized.

Whether reafferent signals from the vestibular semicircular canals are selectively processed during self-generated motion is an issue of continuing controversy. Because it is technically difficult to maintain isolation of a single vestibular receptor cell or primary afferent during active motion, this question has been addressed only at the level of second-order neurons in the vestibular nucleus. Recent experiments in squirrel monkey (Boyle et

\footnotetext{
Received Oct. 16, 2000; revised Dec. 22, 2000; accepted Dec. 22, 2000.

This study was supported by the Medical Research Council of Canada. We thank Drs. D. Guitton, D. Watt, and G. Mandl for many helpful discussions, P. A. Sylvestre, M. Huterer, and A. Dubrovsky for critically reading this manuscript, and M. Drossos, W. Kucharski, J. Knowles, and A. Smith for excellent technical assistance.

Correspondence should be addressed to Kathleen E. Cullen, Aerospace Medical Research Unit, 3655 Drummond Street, Montreal, Quebec, Canada H3G 1Y6. E-mail: cullen@med.mcgill.ca.

Copyright (C) 2001 Society for Neuroscience $0270-6474 / 01 / 212131-12 \$ 15.00 / 0$
}

al., 1996; McCrea et al., 1996, 1999) have shown that a specific subclass of second-order neurons [vestibular-only (VO) neurons] are significantly less sensitive to the head motion generated during active eye-head gaze shifts than during passive whole-body rotations. In contrast, in rhesus monkey, single-unit recordings showed that VO neurons similarly encode head velocity during passive whole-body rotation and combined eye-head pursuit (Khalsa et al., 1987). Unfortunately, the interpretation of these previous studies is limited, because neuronal responses were tested during different voluntary behaviors, i.e., gaze shifts (Boyle et al., 1996; McCrea et al., 1999) or gaze pursuit (Khalsa et al., 1987).

Although the above findings in squirrel monkey are consistent with the hypothesis that an efference copy of the neck motor command selectively suppresses vestibular signals that arise from active head motion, it is also possible that other mechanisms mediate the observed attenuation of VO neuron responses. First, VO neurons could be selectively inhibited by the premotor circuitry that generates gaze shifts. This would account for the apparent discrepancy between previous studies, because the brainstem burst generator is active throughout gaze shifts but is silent during smooth pursuit (Keller, 1974; Cullen and Guitton, 1997a). Therefore, we recorded from the same VO neurons during gaze shifts and gaze pursuit to determine whether vestibular reafferent signals were similarly suppressed. In addition, neuronal responses were characterized immediately after gaze shifts in which the burst generator is silent and the axis of gaze is stable, yet the head is still moving in space (Cullen et al., 1993b; Cullen and Guitton, 1997b). Alternatively, it is possible that the monkey's knowledge of its self-generated head motion attenuates the sensitivity of VO neurons to head velocity during gaze shifts. 
To test this hypothesis, we designed a novel behavioral task in which a head-restrained monkey voluntarily "drove" its head and body together in space by manually rotating a steering wheel. Finally, we investigated whether inputs from the neck muscle proprioceptors might contribute, at least in part, to the attenuation of VO neuron responses during gaze shifts. To date, there has been no clear agreement on how strongly this input influences the activity of VO neurons in alert animals (cat: Fuller 1988; squirrel monkey: McCrea et al., 1999; Gdowski and McCrea, 1999; rhesus: Khalsa et al., 1987, 1988). To address this question, neurons were characterized during passive rotation of the head relative to the body while the monkey was generating minimal neck motor commands.

\section{MATERIALS AND METHODS}

Three rhesus monkeys (Macaca mulatta) were prepared for chronic extracellular recording using aseptic surgical techniques. All experimental protocols were approved by the McGill University Animal Care Committee and were in compliance with the guidelines of the Canadian Council on Animal Care.

Surgical procedures. The surgical techniques were similar to those described previously by Sylvestre and Cullen (1999). Briefly, surgical levels of anesthesia were achieved using isoflurane gas (2-3\% initially) and maintained for the duration of the surgery $(0.8-1.5 \%)$. A dental acrylic implant was fastened to each animal's skull using stainless steel screws. A stainless steel post, which was used to restrain the animal's head, and a stainless steel recording chamber, which was positioned to provide access to the medial vestibular nucleus (posterior and lateral angles of $30^{\circ}$ ), were attached to the implant. In the same procedure, an $18-19 \mathrm{~mm}$ in diameter eye coil (three loops of Teflon-coated stainless steel wire) was implanted in the right eye behind the conjunctiva. After the surgery, buprenorphine $(0.01 \mathrm{mg} / \mathrm{kg}$, i.m.) was used for postoperative analgesia. Animals were given 2 weeks to recover from the surgery before any experiments were performed.

Data acquisition. During the experiments, the monkey was comfortably seated in a primate chair, which was fixed to the suprastructure of a vestibular turntable. The monkey's head was initially restrained during each experiment, and the room was dimly lit. Extracellular single-unit activity was recorded using enamel-insulated tungsten microelectrodes (7-10 $\mathrm{M} \Omega$ impedance; Frederick Haer Co., Bowdoinham, ME) as has been described previously (Sylvestre and Cullen, 1999). The locations of the medial and lateral vestibular nuclei were determined relative to the abducens nucleus, which was identified based on its stereotypical discharge patterns during eye movements (Cullen et al., 1993a; Sylvestre and Cullen, 1999). Gaze and head position were measured using the magnetic search coil technique (Fuchs and Robinson, 1966). Turntable velocity was measured using an angular velocity sensor (Watson Inc.). Unit activity, horizontal and vertical gaze and head positions, target position, and table velocity were recorded on digital audio tape for later playback. The isolation of each unit was carefully reevaluated off-line. During playback, action potentials were discriminated using a windowing circuit (BAK Electronics Inc., Germantown, $\mathrm{MD}$ ) that was manually set to generate a pulse coincident with the rising phase of each action potential. Gaze position, head position, target position, and head velocity signals were low-pass filtered at $250 \mathrm{~Hz}$ (eight-pole Bessel filter) and sampled at $1000 \mathrm{~Hz}$.

Behavioral paradigms. All monkeys were trained to follow a target light for a juice reward. The activity of vestibular neurons was initially recorded with the monkey in the head-restrained condition during voluntary eye movements and passive whole-body rotations. A target light (HeNe laser) was projected, via a system of two galvanometer-controlled mirrors, onto a cylindrical screen located $60 \mathrm{~cm}$ away from the monkey's head. Neuronal responses were recorded during eye movements made to track a target that was (1) stepped between horizontal positions over a range of $\pm 30^{\circ}$ and (2) moved sinusoidally $\left(0.5 \mathrm{~Hz}, 80^{\circ} / \mathrm{sec}\right.$ peak velocity) in the horizontal plane. Neuronal sensitivities to head velocity were tested by passively rotating monkeys about an earth vertical axis $(0.5 \mathrm{~Hz}$, $80 \%$ sec peak velocity) in the dark [ passive whole-body rotation ( $\mathrm{pWBR}$ )] and while they cancelled their vestibulo-ocular reflex by fixating a target that moved with the vestibular turntable (pWBRc). Target and turntable motion and on-line data displays were controlled by a UNIX-based real-time data acquisition system (REX; Hays et al., 1982).

After a neuron was fully characterized in the head-restrained condi- tion, the monkey's head was slowly and carefully released. Once released, the monkey was free to rotate its head through the natural range of motion in the yaw (horizontal), pitch (vertical), and roll (torsional) axes. The response of the same neuron was then recorded during the voluntary horizontal head movements made during combined eye-head gaze shifts (15-65 in amplitude) and combined eye-head gaze pursuit of a sinusoidal target $\left(0.5 \mathrm{~Hz}, 80^{\circ} / \mathrm{sec}\right.$ peak velocity). Neuronal responses to combined passive and active head motion were tested by passively rotating $\left(0.5 \mathrm{~Hz}, 80^{\circ} / \mathrm{sec}\right.$ peak velocity) monkeys in the head-unrestrained condition, such that they could simultaneously generate voluntary headon-body movements. We analyzed those intervals in which head motion velocity differed from turntable velocity by $>10^{\circ} / \mathrm{sec}$. The active component of head motion was calculated by subtracting the turntable velocity from the head-in-space velocity. The passive component of head motion was the velocity of the passive turntable rotation. This analysis assumes that the vestibulo-collic reflex (VCR) response is minimal in rhesus monkeys, which is consistent with our preliminary data (our unpublished observations). Finally, to investigate the influence of neck proprioceptive inputs on neural discharges, two different paradigms were used: (1) the monkey's head was held stationary relative to the earth while its body was passively rotated below $\left(0.5 \mathrm{~Hz}, 80^{\circ} / \mathrm{sec}\right.$ peak velocity), and (2) the experimenter manually rotated the monkey's head to induce rapid motion of the head relative to a stationary body. In the first of these two paradigms, the torque produced against the head restraint was measured using a reaction torque transducer (Sensotec, Columbus, $\mathrm{OH}$ ).

Neuronal responses to voluntary head-in-space motion generated by behaviors that did not involve activation of the neck musculature were characterized using one of two head-restrained paradigms. In the first, monkeys fixated a light-emitting diode (LED) target that was attached to the turntable. When the LED began to flash, the monkey depressed a switch initiating a constant velocity-direction rotation of the turntable $\left(40^{\circ} / \mathrm{sec}\right)$. In the second, monkeys were trained to operate a steering wheel that controlled the rotation of the turntable on which they were seated to track a laser or a food target. During this latter "driving paradigm," the position of the steering wheel was fed into the turntable servo, which in turn controlled the position of the turntable. Thus, monkeys controlled the initiation of the movement and also the rotational velocity of the turntable, via the speed at which they rotated the steering wheel.

Analysis of neuron discharges. Before analysis, recorded gaze and head position signals were digitally filtered at $125 \mathrm{~Hz}$. Eye position was calculated from the difference between gaze and head position signals. Gaze, eye, and head position signals were digitally differentiated to produce velocity signals. The neural discharge was represented using a spike density function in which a Gaussian function was convolved with the spike train (SD of $5 \mathrm{msec}$ for saccades and gaze shifts and $10 \mathrm{msec}$ for the remainder of the paradigms) (Cullen et al., 1996). Saccade and gaze shift onsets and offsets were defined using a $\pm 20^{\circ} / \mathrm{sec}$ gaze velocity criterion. Subsequent analysis was performed using custom algorithms (Matlab; MathWorks Inc., Natick, MA). Statistical significance was determined using a paired Student's $t$ test.

In this study, we only present data from neurons that were not sensitive to eye position during ocular fixation and eye position or velocity during smooth pursuit. To verify that a neuron was unresponsive to eye position and/or velocity, we analyzed periods of steady fixation and saccade-free smooth pursuit using a multiple regression analysis (Roy and Cullen, 1998). A least-squared regression analysis was then used to determine the phase shift of each unit relative to head velocity, resting discharge (bias, spikes per second), and head velocity sensitivity $\left[g_{\text {pWBR }}\right.$ (spikes per second)/(degrees per second)]. This analysis was done during $\mathrm{pWBR}$ and pWBRc to obtain two estimates of head velocity sensitivity of a neuron. Only unit data from periods of slow-phase vestibular nystagmus (pWBR) or steady fixation (pWBRc) that occurred between quick phases of vestibular nystagmus and/or saccades were included in the analysis.

Confirmation of neuron isolation. To confirm that isolation of the same neuron was maintained before and after the head-restrained-headunrestrained transition, resting discharge rates were compared. Values were not significantly different before and after head release on a neuronby-neuron basis (population mean, $61 \pm 33$ vs $64 \pm 33$ spikes/sec, respectively; $\left.R^{2}=0.9 ; p>0.8\right)$. In addition, the $\mathrm{pWBRc}$ paradigm was repeated for the majority $(77 \%)$ of neurons after head release, and the neuronal modulation was found to be comparable with that observed during the initial head-restrained characterization [mean head velocity sensitivity, $0.53 \pm 0.24$ vs $0.50 \pm 0.25($ spikes $/ \mathrm{sec}) /\left({ }^{\circ} / \mathrm{sec}\right)$, respectively; $\left.R^{2}=0.86 ; p>0.6\right]$. 


\section{RESULTS}

\section{Vestibular-only neurons: head-restrained condition}

A distinct population of vestibular nuclei neurons, VO neurons, are known to receive direct monosynaptic projections from vestibular nerve afferents (Scudder and Fuchs, 1992; Cullen and McCrea, 1993; McCrea et al., 1999). To date, VO neurons have been well characterized in head-restrained monkeys (Fuchs and Kimm, 1975; Keller and Kamath, 1975; Tomlinson and Robinson, 1984; Scudder and Fuchs, 1992; Cullen and McCrea, 1993); their firing frequency is modulated by head-in-space motion during whole-body rotation but not by eye-in-head motion. An example of VO neuron discharge is illustrated in Figure 1. During pWBR about an earth vertical axis, the neuron was strongly modulated in response to ipsilaterally directed head velocity [head velocity sensitivity, 0.62 (spikes/sec) $/(\% / \mathrm{sec})$ ] (Fig. 1A). Because the pWBR paradigm elicited a compensatory eye motion response [i.e., the vestibulo-ocular reflex (VOR)], each neuron was also characterized during passive whole-body rotation while the monkey suppressed its VOR by fixating a visual target that moved with its head (pWBRc) (Fig. 1B). This neuron was representative of the cells in our sample $(n=40)$ in that its head velocity sensitivity during pWBRc was the same as during pWBR [sample mean head velocity sensitivity, $0.52 \pm 0.24( \pm \mathrm{SD})$ and $0.53 \pm 0.24$ (spikes/sec)/(\%/sec), respectively]. Moreover, all neurons were unresponsive to eye position during steady fixation (Fig. 1C), eye motion during saccades (Fig. 1C, arrows), and smooth pursuit (Fig. 1D). Depending on whether their activity increased during ipsilaterally $(n=23)$ or contralaterally $(n=17)$ directed passive whole-body rotation, neurons were further classified as type I or type II, respectively. For the purpose of this paper, type I and II neurons were considered collectively, because they encoded similar signals during each behavioral task.

\section{Vestibular-only neurons: voluntary head-on-body motion}

The sensitivity of each VO neuron to voluntary head motion was also characterized during combined eye-head gaze shifts. The monkey's head was released from its restraint, allowing rotation through the natural range of motion in all three axes. During the critical transition between the head-restrained and headunrestrained conditions, the waveform of the action potential of each neuron was carefully monitored to ensure that the cell remained undamaged and well isolated (see Materials and Methods). Our example neuron was typical of all neurons tested in that it was poorly modulated by voluntary head motion during small $\left(25-35^{\circ}\right)$ and large $\left(55-65^{\circ}\right)$ horizontal gaze shifts (Fig. $2 A$, filled arrows, left and right panels, respectively). For each cell, we determined whether a model (Fig. $1 A$, thick trace) based on the head velocity sensitivity of the neuron during passive whole-body rotation could predict the firing rate of the neuron during combined eye-head gaze shifts. The model is given by the following equation:

$$
f r=\text { bias }+\left(g_{\mathrm{pWBR}} * \text { head velocity }\right) \quad(\mathrm{pWBR} \text { prediction })
$$

where $f r$ is the firing rate, and $g_{\mathrm{pWBR}}$ is the head velocity sensitivity of the neuron during passive whole-body rotation. This model consistently overestimated the discharge of the neuron during active head-in-space motion (Fig. 2A, pWBR prediction, thick traces). To quantify this observation, we determined the best estimate of head velocity sensitivity of each neuron $\left(g_{\text {est }}\right)$ during gaze shifts (range, $15-65^{\circ}$ ) using the following equation:

$$
\left.f r=\text { bias }+\left(g_{\text {est }} * \text { head velocity }\right) \quad \text { (estimate }\right)
$$

VO neurons were less modulated for the voluntary head-on-body movements made during gaze shifts than during passive wholebody rotation (Fig. $2 A$, compare $p W B R$ prediction with estimate). The mean head velocity sensitivity of our sample of neurons was significantly reduced compared with that observed during $\mathrm{pWBR}$ $[0.17 \pm 0.16$ vs $0.53 \pm 0.24$ (spikes $\left./ \mathrm{sec}) /\left({ }^{\circ} / \mathrm{sec}\right) ; p<0.005\right]$, in agreement with previous studies in rhesus monkey (Roy and Cullen, 1999) and squirrel monkey (Boyle et al., 1996; McCrea et al., 1996, 1999).

Here and for all subsequent tasks (see below) during which VO neurons were tested, head velocity sensitivity of each cell was normalized relative to passive whole-body rotation ( $\mathrm{pWBR}$ ) to facilitate comparison (normalized sensitivity, $g_{\text {est }}$ for a given task $\left(g_{\mathrm{pWBR}}\right)$. For example, during gaze shifts, the normalized head velocity sensitivity of VO neurons was $0.17 \pm 0.16 / 0.53 \pm$ $0.24=0.32 \pm 0.35(\mathrm{spikes} / \mathrm{sec}) /(\% / \mathrm{sec})$, corresponding to an attenuation of $68 \%$.

Figure $2 B$ illustrates that the attenuation of neural sensitivities to head velocity that we observed during gaze shifts was independent of gaze shift amplitude. For each neuron, gaze shifts were sorted into separate data sets, each spanning $10^{\circ}$ and containing at least 10 and generally 15 or more examples. The head velocity sensitivities were estimated separately for each amplitude range. For our entire sample of VO neurons, the attenuation of head velocity sensitivity was not significantly different for large gaze shifts than for small ones [e.g., normalized $g_{\text {est }}, 0.36 \pm 0.33$ (spikes/sec) $/\left({ }^{\circ} / \mathrm{sec}\right)$ for $55-65^{\circ}$ vs $0.28 \pm 0.36($ spikes $/ \mathrm{sec}) /\left({ }^{\circ} / \mathrm{sec}\right)$ for $\left.15-25^{\circ}\right) ; p>0.2$ ] and was always significant relative to $\mathrm{pWBR}$ $(p<0.005)$.

To determine whether the same neurons demonstrated similar attenuation during all voluntary motion of the head on the neck, neural responses were characterized during (1) voluntary head movements made when gaze was immobile and (2) voluntary head movements made during combined eye-head gaze pursuit. To address whether VO neuron responses to head motion were attenuated during the period that immediately followed a gaze shift in which the head was still moving but gaze was immobile (Fig. 2A, open arrows), a quantitative analysis was performed. The $\mathrm{pWBR}$ prediction provided a poor estimate of VO neuron activity during this interval (thick traces). We obtained an estimate of head velocity sensitivity ( $g_{\text {est }}$ ) over the interval of $10-80$ msec that immediately followed each gaze shift and found that the mean calculated attenuation in neural modulation was comparable with that observed during gaze shifts [normalized $g_{\text {est }}$, $0.35 \pm 0.3$ (spikes $\left./ \mathrm{sec}) /\left({ }^{\circ} / \mathrm{sec}\right) ; p>0.6\right]$. Figure $2 C$ illustrates the attenuation in head velocity sensitivities that we observed immediately after gaze shifts for our population of VO neurons. As was the case during gaze shifts, the level of attenuation did not vary systematically with gaze shift amplitude $(p>0.5)$. Furthermore, for our entire sample of VO neurons, attenuation was comparable during the postgaze shift interval and the gaze shift itself for all amplitudes $(p>0.9)$ (Fig. 2, compare $B, C)$.

We characterized VO neuron responses to head motion during combined eye-head gaze pursuit and obtained an analogous result (Fig. $3 A)$. All neurons tested $(n=31)$ were less sensitive to head-in-space (or head-on-body) motion during gaze pursuit [normalized $g_{\text {est }}, 0.32 \pm 0.44$ (spikes $\left.\left./ \mathrm{sec}\right) /(\% / \mathrm{sec})\right]$ than during passive whole-body rotation $(p<0.005)$. This is illustrated for our example neuron in which the pWBR prediction (Fig. 3A, thick trace) consistently overestimated the modulation of the neuron during gaze pursuit. The mean peak head movement velocities generated in this task were significantly less than those 
A Passive whole-body rotation

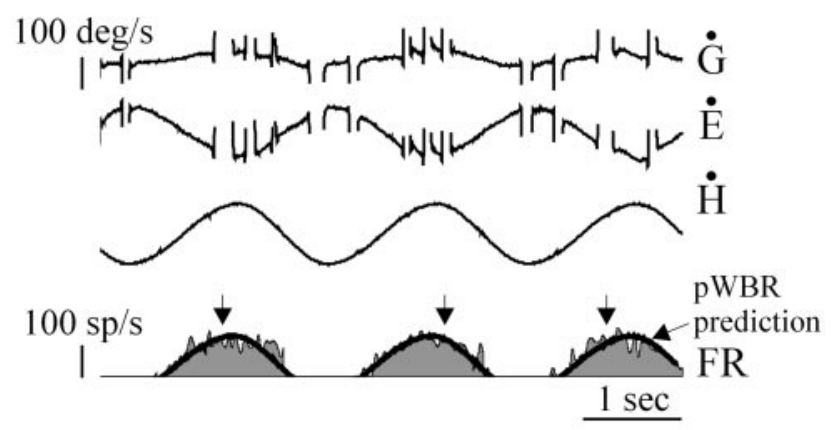

\section{B Passive whole-body rotation during cancellation}

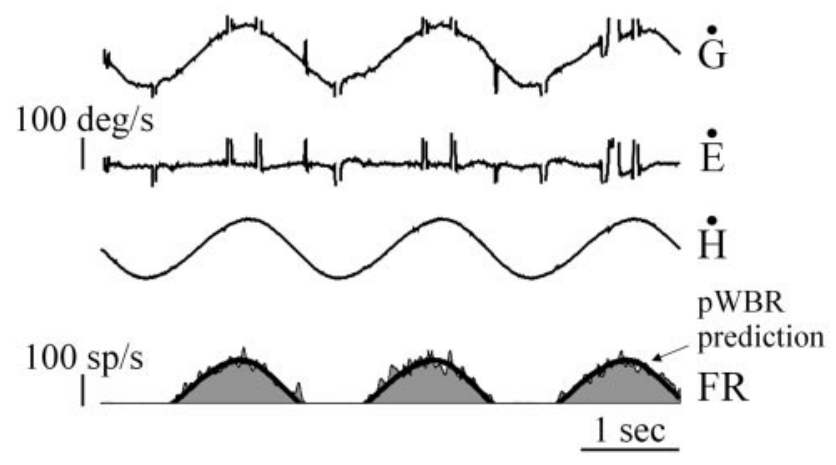

C Saccades
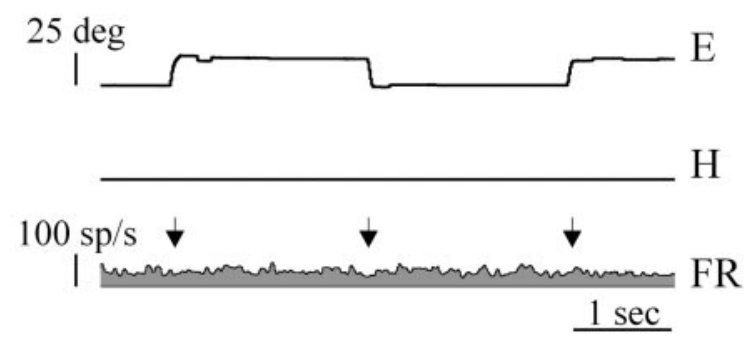

\section{Smooth Pursuit}

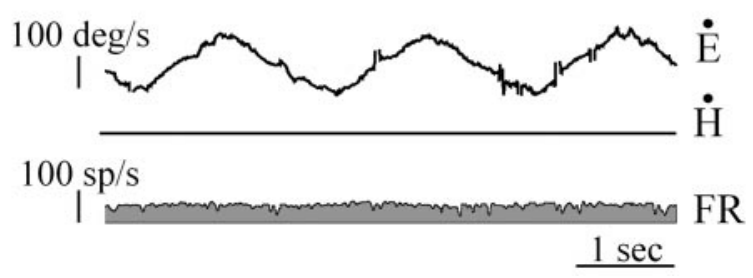

Figure 1. Activity of an example VO neuron (unit 79_5) during the head-restrained condition. $A, B$, Passive whole-body rotation was used to characterize the response of the neuron to head movements during VOR in the dark $(A)$ and head movements while the monkey cancelled its VOR by fixating a target that moved with the table $(B)$. A model based on head-restrained head movement sensitivities during VOR in the dark ( $p$ WBR prediction, thick trace) is superimposed on the firing rate traces. generated during gaze shifts larger than $35^{\circ}$ (Fig. $3 B$ ) and were much less stereotyped. Nevertheless, the estimated head velocity sensitivities during gaze pursuit were comparable with those observed during gaze shifts on a neuron-by-neuron basis $\left(H_{0}\right.$, slope of $1 ; p>0.9$ ) (Fig. 4).

Figure 5 summarizes the head velocity information carried by VO neurons during passive whole-body rotation paradigms versus self-generated head motion resulting from activation of the neck musculature. We found that the head velocity signal carried by our population of neurons was similarly attenuated for all behaviors in which head-in-space motion resulted from active motion of the head-on-body (gray columns). Furthermore, the observed attenuation was not dependent on whether the behavioral goal was to redirect gaze (gaze shifts and gaze pursuit) or stabilize gaze in space (i.e., in the interval after gaze shifts). VO neuron responses were similarly decreased during and immediately after gaze shifts [normalized $g_{\text {est }}$ averaged across all amplitudes, $0.32 \pm$ 0.33 vs $0.35 \pm 0.3($ spikes $/ \mathrm{sec}) /(\% / \mathrm{sec})]$ and gaze pursuit [normalized $g_{\text {est }}, 0.32 \pm 0.44$ (spikes/sec) $\left./(\% / \mathrm{sec})\right]$. In contrast, when gaze was redirected during passive whole-body rotation to cancel the VOR, VO neurons showed little or no attenuation in their head velocity sensitivity [black column; normalized $g_{\text {pW BRc }}, 0.97 \pm 0.12$ (spikes/sec) $/(\% / \mathrm{sec})]$. Thus, the discharge of VO neurons depended on whether head-in-space motion resulted from an active head-on-body movement and not the monkey's gaze strategy (i.e., to stabilize or redirect gaze). The implications of this differential processing of head velocity information will be considered in Discussion.

\section{Vestibular-only neurons: simultaneous voluntary and passive head motion}

We next addressed whether VO neurons selectively encode vestibular inputs that arise from external sources (i.e., passively applied motions) when the vestibular system is simultaneously stimulated by passive and self-generated head motion. Neurons were recorded while the monkey generated voluntary head movements on its body (Fig. 6A, dashed arrow in schema) while undergoing passive whole-body rotation (Fig. 6A, filled arrow in schema). During this paradigm, the head-in-space movement $\left(\dot{\mathrm{H}}_{\mathrm{S}}\right)$ is the sum of the passive whole-body rotation $\left(\dot{\mathrm{B}}_{\mathrm{S}}\right)$ and the voluntarily generated head-on-body motion $\left(\dot{\mathrm{H}}_{\mathrm{B}}\right)$. Remarkably, our example neuron responded robustly to only the component of head-in-space motion produced by passive rotation of the body (Fig. $6 A, \dot{B}_{S}$ prediction, thin dark trace and $\square$ in bottom left panel). This finding is in agreement with studies in squirrel monkey (Boyle et al., 1996; McCrea et al., 1999). In contrast, the neuronal response to the component of head-in-space motion generated by the monkey's own voluntary head-on-body movements was relatively weak or negligible (Fig. $6 A, \dot{H}_{S}$ prediction, thick dark trace and in bottom left panel). For the sample of neurons $(n=24)$, responses to the voluntary component of head-on-body motion were significantly reduced when gaze was redirected [normalized $g_{\text {vol }}, 0.33 \pm 0.33$ (spikes $\left./ \mathrm{sec}\right) /(\%$ sec) $]$ (Fig. $6 B$, black column) or stabilized [normalized $g_{\text {vol }}, 0.33 \pm 0.36$ (spikes $\left.\left./ \mathrm{sec}\right) /(\% / \mathrm{sec})\right]$ (Fig. $6 B$, gray column), whereas responses to the passive component of

$\leftarrow$

$C, D$, The neuron was unresponsive to eye movements during saccades (arrows in $C$ ) and smooth pursuit $(D)$. Note that neurons were also unresponsive to vestibular quick phases (arrows in $A$ ). Traces directed upward are in the ipsilateral direction. $E$, Eye position; $H$, head position; $\dot{E}$, eye-in-head velocity; $\dot{H}$, head velocity; $\dot{G}$, gaze velocity $(\dot{E}+\dot{H}) ; F R$, firing rate. 

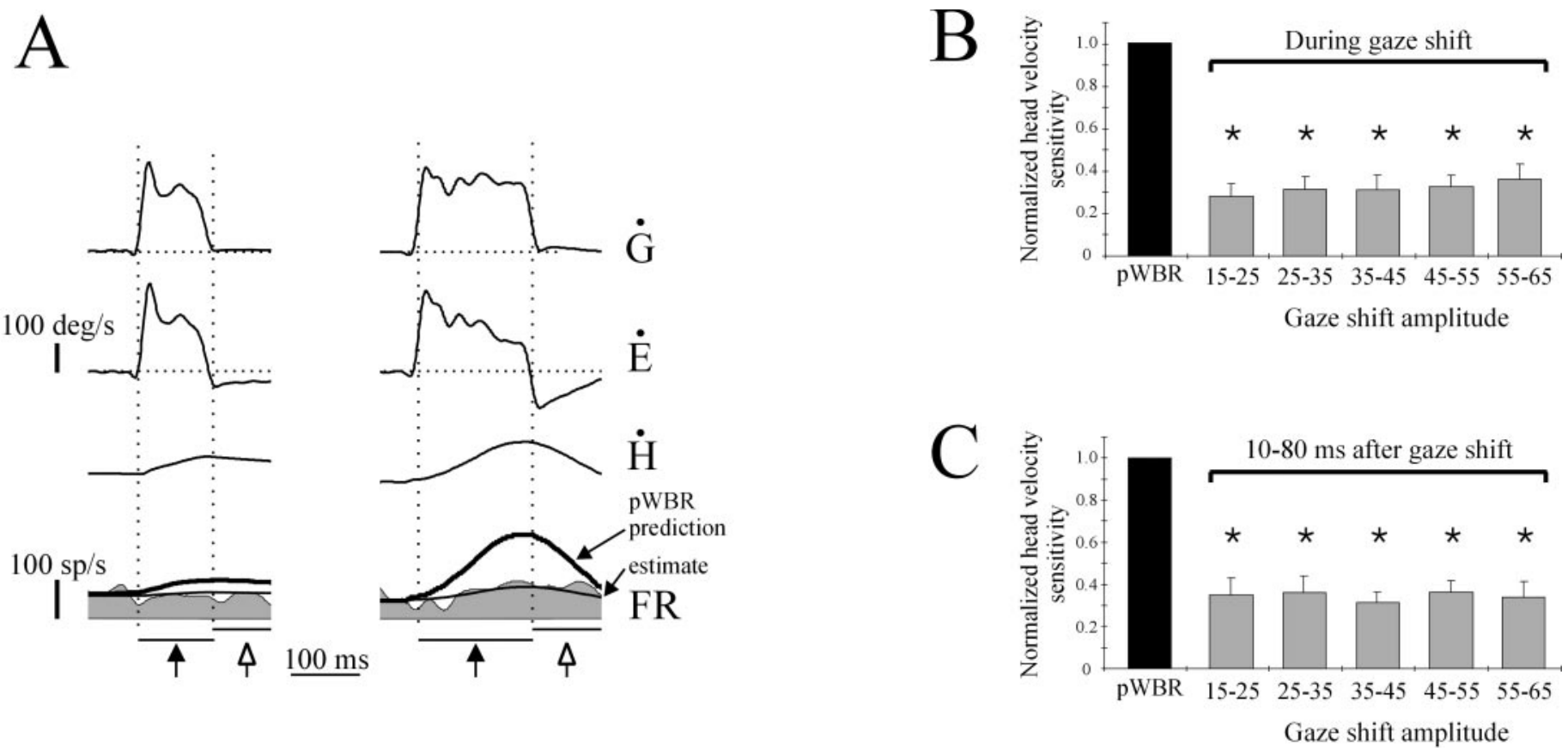

Figure 2. Activity of an example VO neuron (unit 79_5) during and after voluntary combined eye-head gaze shifts. $A$, Superimposed on the firing rate are fits for the pWBR prediction and the estimate of the head velocity sensitivity. The pWBR prediction overestimated the discharge of this neuron during small (middle panel) and large (right panel) amplitude gaze shifts. Dotted vertical lines indicate the onset and offset of gaze shifts using a $\pm 20^{\circ} / \mathrm{sec}$ criterion. Two time intervals are denoted: the duration of the gaze shift (large filled arrows) and 10-80 msec immediately after the gaze shift (large open arrows). $B$, During gaze shifts, the head velocity sensitivity of our sample of VO neurons was similarly attenuated for all gaze shift amplitudes from 15 to $65^{\circ}$ (gray columns), and responses were significantly $(p<0.005)$ smaller than those resulting from pWBR (black column). $C$, Comparable attenuation was observed in the postgaze shift interval (gray columns). Error bars show SEM. Abbreviations as in Figure 1.
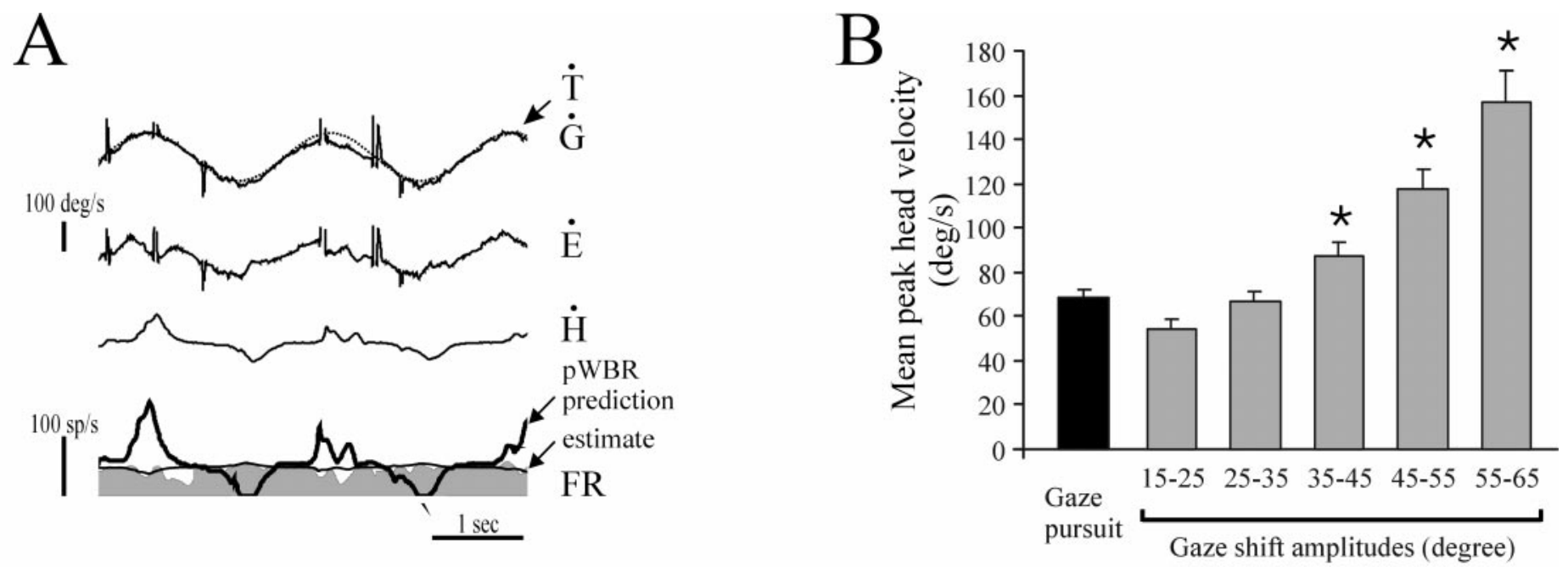

Figure 3. Responses of a typical VO neuron (unit 79 5) to voluntary head-on-body motion during combined eye-head gaze pursuit. $A$, The response of the unit to self-generated head motion was reduced compared with that predicted by the sensitivity of the neuron to passive whole-body rotation (compare $p W B R$ prediction and estimate). B. Comparison of head velocities generated during gaze shifts and gaze pursuit. The mean peak head velocities generated during gaze shifts $\left(>35^{\circ}\right.$ in amplitude; gray columns) were significantly larger than those generated during gaze pursuit (black column). $\dot{T}$, Target velocity. All other abbreviations as in Figure 1.

the motion were not attenuated, regardless of monkey's gaze strategy [normalized $g_{\text {pass }}, 0.98 \pm 0.12$ and $0.95 \pm 0.23$ (spikes/ $\mathrm{sec}) /(\% \mathrm{sec})$ gaze redirected and stabilized, respectively] (Fig. $6 B$, white columns). Thus, the vestibular afferent input to VO neurons was not cancelled in its entirety (i.e., gated out) during selfgenerated head motion; vestibular afferent signals related to the voluntary head-on-body motion were effectively suppressed, but neurons continued to respond to unexpected perturbations of the head.

\section{Vestibular-pause neurons}

To emphasize the implications of the above findings, we recorded from an additional 10 neurons termed vestibular-pause (V-pause) neurons, which have been well characterized in head-restrained 


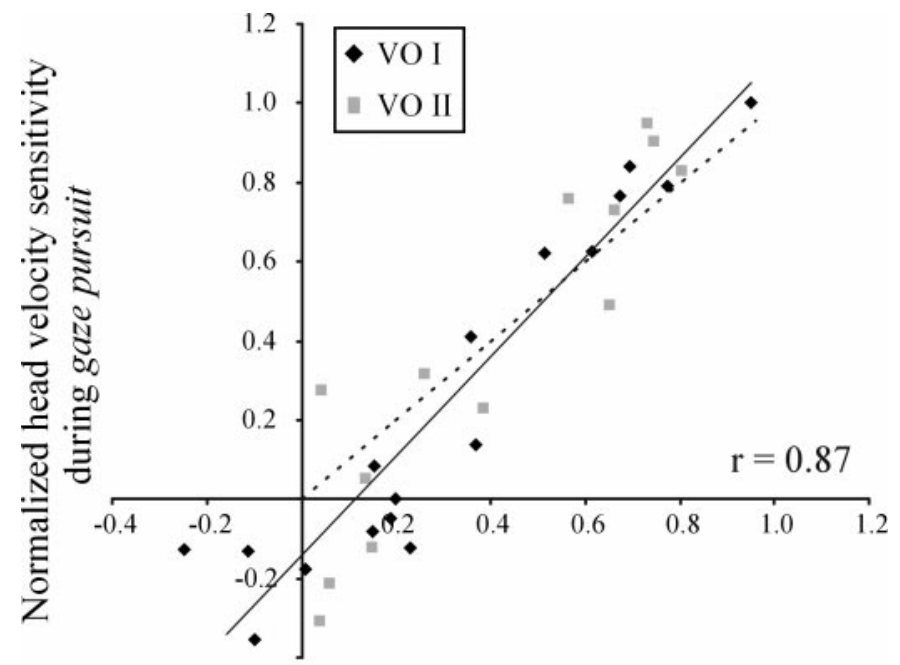

Normalized head velocity sensitivity
during gaze shifts

Figure 4. VO neuron responses during gaze shifts and gaze pursuit. Despite the differences in peak head velocities and type of eye motion generated during the two tasks, the normalized head velocity sensitivities were not significantly different. Comparable trends were found for type I $(\diamond)$ and type II (橉) VO neurons. Dashed line represents unity.

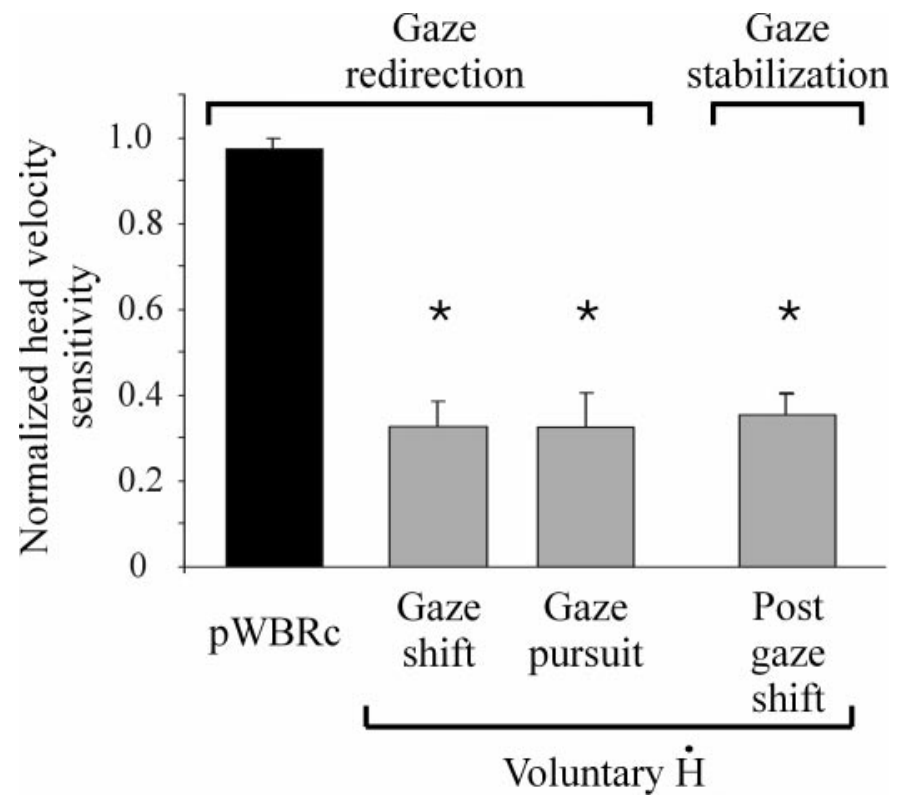

Figure 5. During self-generated head-on-body motion, the suppression of the head velocity sensitivity was not dependent on whether the monkey was stabilizing or redirecting its gaze. During the pWBRc paradigm (black column) in which the monkey was redirecting its gaze, the neuronal responses were not significantly different from those observed during $\mathrm{pWBR}$, yet when gaze was redirected during gaze shifts and gaze pursuit (gray columns), neuronal responses were significantly attenuated. VO neurons response were selectively attenuated during active motion of the head-on-body: gaze shifts, gaze pursuit, and the period immediately after the gaze shifts (rightmost column) in which gaze was immobile but the head continued to move. Error bars show SEM.

animals (Fuchs and Kimm, 1975; Keller and Kamath, 1975; Scudder and Fuchs, 1992; McCrea et al., 1999). V-pause neurons differ from VO neurons only in that they stop firing (pause) for saccades and vestibular quick phases (Fig. 7 arrows, $A$ and $B$, respectively). Like VO neurons, V-pause neurons are not sensitive to eye position (Fig. $7 A$ ) or eye velocity during smooth pursuit (data not shown) and are strongly modulated by head-inspace motion during pWBR (Fig. 7B). Moreover, their responses during pWBR and cancellation of the VOR (pWBRc) are comparable [head velocity sensitivities, $0.90 \pm 0.60$ and $0.87 \pm 0.47$ (spikes/sec)/(\%/sec)]. V-pause neurons further resembled VO neurons in that their vestibular responses to active head-on-body movements immediately after gaze shifts (Fig. 7C, open arrows) and during gaze pursuit were similarly attenuated [normalized $\mathrm{g}_{\text {est }}, 0.47 \pm 0.23$ vs $0.56 \pm 0.19$ (spikes/sec) $/(\% / \mathrm{sec})$, respectively; $p>0.1$. Figure $7 C$ shows that the normalized head velocity sensitivities estimated for these two tasks were well correlated for the population of neurons (right panel) $\left(H_{0}\right.$, slope of $\left.1 ; p>0.2\right)$. However, during gaze shifts, V-pause neurons paused (Fig. $7 C$, filled arrows) as they did during head-restrained saccades. Accordingly, the normalized head velocity sensitivity of all V-pause cells was nearly zero [normalized $\mathrm{g}_{\text {est }}, 0.12 \pm 0.14$ (spikes/sec) $/(\%$ $\mathrm{sec})]$ and was poorly correlated with vestibular sensitivities measured during other self-generated movements of the head-onbody. Head velocity sensitivities of V-pause neurons were estimated using a model that included an eye velocity term, as well as a bias term and a head velocity term. In addition, the neural responses of V-pause neurons during simultaneous stimulation with passive and voluntary head motion were similar to those of VO neurons when the monkey's goal was to either stabilize its gaze or redirect its gaze using slow eye movements. Otherwise, during rapid eye movements, the neurons paused in activity. Thus, V-pause neurons differ from vestibular-only neurons in that they appear to receive an additional source of inhibition from the saccadic premotor circuitry during gaze shifts, as well as during saccades and vestibular quick phases. The responses of V-pause neurons were comparable with VO neurons, except during rapid eye movements, for the remainder of the tasks in this study.

\section{Self-generated motion of the head and body in space}

To determine whether the monkey's knowledge of its selfgenerated head motion attenuates the sensitivity of VO neurons to head velocity, we characterized neural activity during two voluntary tasks in which the head and body moved together relative to space. In the first task (Fig. $8 A$, schema), monkeys were trained to depress a switch at the appearance of a light cue. By pushing the switch, the monkey initiated a vestibular turntable rotation of a constant velocity, direction, and duration, after which it received a juice reward. The example neuron was typical of the three neurons tested in that it was strongly activated by the resultant head motion; its activity could be reliably predicted by its head velocity sensitivity during passive whole-body rotation (Fig. 8A, $p W B R$ prediction, thick trace). During this task, the monkey anticipated the head movement, because it initiated the motion of the turntable. However, it is arguable whether the resultant head movement was truly voluntary, because the monkey had no control over the actual trajectory of table motion.

To address this issue, we designed a second task in which the monkey directly controlled the velocity and direction of the vestibular turntable. Monkeys drove their own body motion by rotating a steering wheel connected to the motor controller of the vestibular turntable (Fig. 8B, schema). Each animal was well trained in this task and would generate accurate head-in-space motion to align its head-body position with either a laser target [laser task (Fig. 8B)] or a food reward (banana task; data not 

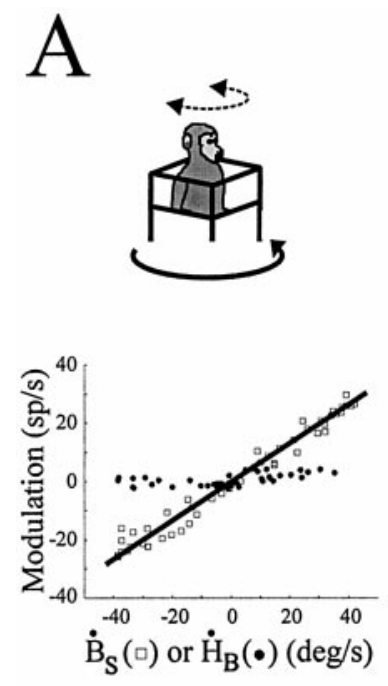
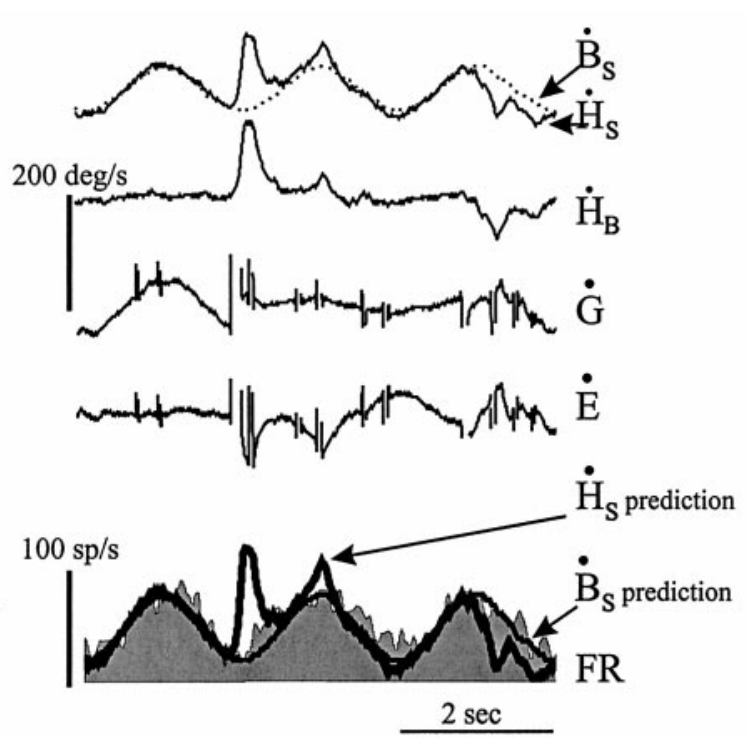

B

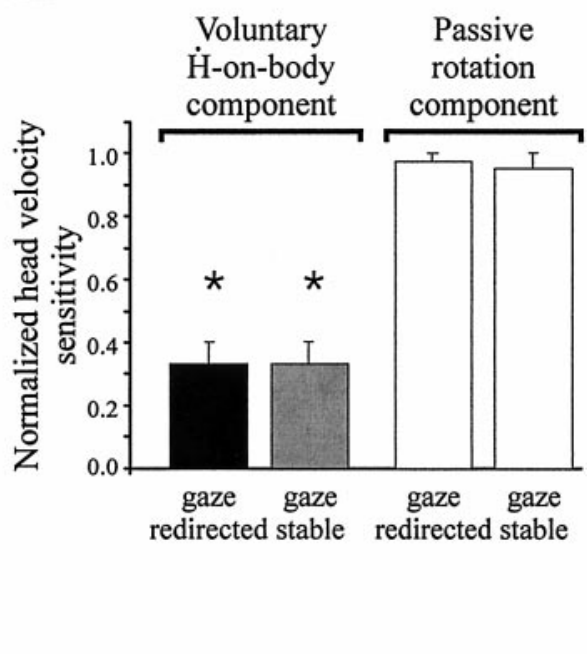

Figure 6. VO neuron responses to combined voluntary and passive head-in-space motion. $A$, Head-unrestrained monkeys generated voluntary head-on-body movements (dashed arrow in schema) while being passively rotated by the vestibular turntable (thick arrow in schema). Head-in-space movement $\left(\dot{\mathrm{H}}_{\mathrm{S}}\right)$ is the sum of the body-in-space motion $\left(\dot{\mathrm{B}}_{\mathrm{S}}\right)$ generated by passive rotation and voluntary head-on-body movements $\left(\dot{\mathrm{B}}_{\mathrm{B}}\right)$. The modulation of the example neuron (unit 79_5) was well correlated with the passive body-in-space motion $\left(\dot{B}_{S}\right.$ prediction, thin dark trace) but was poorly related to the voluntary head-on-body component of head-in-space motion $\left(\dot{H}_{S}\right.$ prediction, thick dark trace). Bottom left panel, Modulation in response to passive body-in-space motion $(\square)$ and to voluntary head-on-body motion during combined stimulation (๑). $B$, The response to the voluntary component of head-in-space motion was significantly attenuated when the monkey was either redirecting (black column) or stabilizing ( gray column) its gaze. In contrast, the neurons showed no attenuation in response to the passive rotation component (white columns). Error bars show SEM. All other definitions as in Figure 1.

shown). Thus, the trajectory of the head motion was the direct result of a goal-directed action taken by the monkey. All neurons tested $(n=15)$ were strongly modulated by the resultant voluntary head-in-space motion [normalized $g_{\text {est }}, 1.04 \pm 0.18$ and $1.05 \pm 0.16$ (spikes/sec) $/(\% / \mathrm{sec})$, laser and banana task, respectively]. Moreover, the activity of each neuron was predicted well by a model based on the modulation of the neuron during passive whole-body rotation (Fig. $8 B, p W B R$ prediction, thick trace). Together, these results indicate that vestibular-related modulation was not attenuated by the knowledge of self-generated motion of the head relative to space and that the specific motor command (i.e., activation of the neck musculature) used to produce the behavior must be considered.

\section{Effect of neck afferent activation}

It is known that in decerebrate animals neck muscle spindle afferents influence the activity of vestibular nuclei neurons (Boyle and Pompeiano, 1981; Anastasopoulos and Mergner, 1982; Wilson, 1991) via a disynaptic pathway (Sato et al., 1997). It is conceivable that in alert monkeys neck afferent inputs contribute to the suppression of VO neuron responses during and after gaze shifts (Fig. $2 A$ ) and during gaze pursuit (Fig. $3 A$ ). To address this possibility, we tested neurons during two experiments in which the head was rotated relative to the body. It is likely that the passive neck rotation resulted in even greater activation of neck proprioceptors than did active head-on-body rotations, because gamma motoneurons are more active during passive than active movements (Prochazka et al., 1987), and, in turn, the sensitivity of muscle spindles to passive neck rotation is likely to be greater (Hulliger et al., 1977).

In the first experiment, the monkey's head was held stationary relative to space and its body was rotated beneath it (Fig. $9 A$, filled arrow in schema). The example VO neuron was representative of our sample in that it vigorously responded to passive whole-body rotation (Fig, $1 A$ ) but was unresponsive to passive rotation of the body under the neck (Fig. 9A, bottom left panel, compare with $\square)$. The activity of the neuron was well described by its spontaneous discharge rate (Fig. $9 A, \dot{H}_{B}$ prediction, thick trace) and poorly predicted by a model that would be consistent with neck afferent inputs suppressing vestibular responses during voluntary head-on-body motion (Fig. $9 A, \dot{B}_{S}$ prediction, thin trace). Neck rotation sensitivities were negligible [normalized sample mean, $0.12 \pm 0.18$ (spikes/sec) $/(\% / \mathrm{sec})]$ (Fig. $9 B$, white column) for all neurons tested $(n=15)$ and thus did not significantly contribute to the attenuation that was observed during self-generated head motion (Fig. 9B, gray columns). The torque produced by the monkey against the head restraint was concurrently measured and found to be small (less than $\pm 0.5 \mathrm{Nm}$ ) compared with that produced when monkeys oriented to food targets (torque of more than $\pm 3.5 \mathrm{Nm}$ ). Thus, the neck motor commands, and by extension neck motor efference copy signals, generated by the monkeys were minimal during these passive rotations.

The influence of neck afferent inputs was further investigated using a paradigm during which the experimenter rapidly rotated the animal's head on its neck (Fig. 10A, hand in schema). The passively elicited head-on-body movements had head-in-space trajectories and velocities comparable with those generated during large voluntary gaze shifts (mean peak head velocity, $167 \pm 63$ vs $153 \pm 55 \%$ sec for $55-65^{\circ}$ gaze shifts). Torque was not measured during this paradigm, but the analysis was restricted to intervals in which monkeys exhibited little resistance to rotation. The neuronal firing rate of the example neuron was well predicted by the head velocity sensitivities during passive head-on-body rotation (Fig. 10A, pWBR prediction, thick trace), indicating that the passive activation of the neck muscle spindles did not alter the 
A saccades

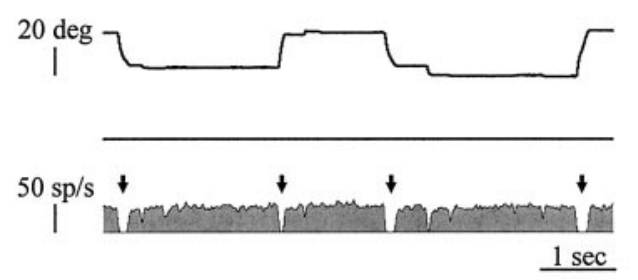

B

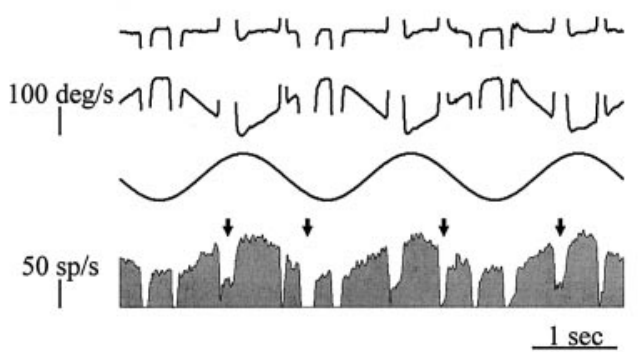

FR
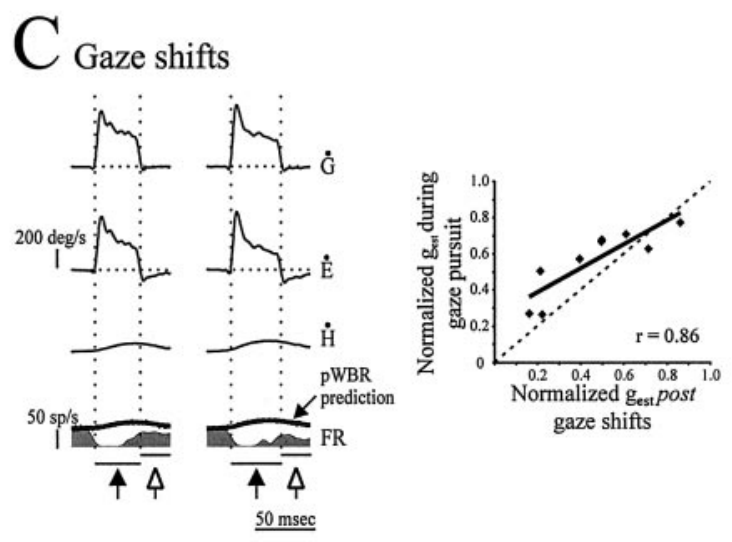

gaze shifts

Figure 7. Activity of an example V-pause neuron (unit b44_1) during head-restrained and head-unrestrained tasks. $A, \mathrm{~V}$-pause neurons paused (stop firing) during saccades (arrows) and were not sensitive to eye position. $B$, During pWBR, V-pause neurons were strongly modulated and paused for vestibular quick phases in both directions (arrows). $C$, V-pause neurons paused during gaze shifts (filled arrows) and responded with an attenuated sensitivity to head motion immediately after gaze shifts (open arrows, $p W B R$ prediction). Bottom left panel, The normalized head velocity sensitivities during the postgaze shift period and during gaze pursuit were comparable for our sample of V-pause neurons (slope of 0.70$)$. Definitions as in Figure 1.

sensitivity of VO neurons to head-in-space motion [Fig. 10 $\mathrm{A}$, bottom left panel, compare the responses to passive neck motion (O) with responses to passive head motion ( $\square$ )]. Furthermore, for the neurons tested $(n=23)$, the neural head velocity sensitivities during passive head-on-body rotations were similar to those measured during passive whole-body rotations, regardless of whether the monkey was redirecting or stabilizing its gaze (Fig. 10B, compare black columns with gray columns).

Likewise, passive activation of neck proprioceptors did not influence the discharges of V-pause neurons. When the monkey's head was held stationary and its body was rotated beneath it, neuronal sensitivities to neck velocity were negligible [sample of

A
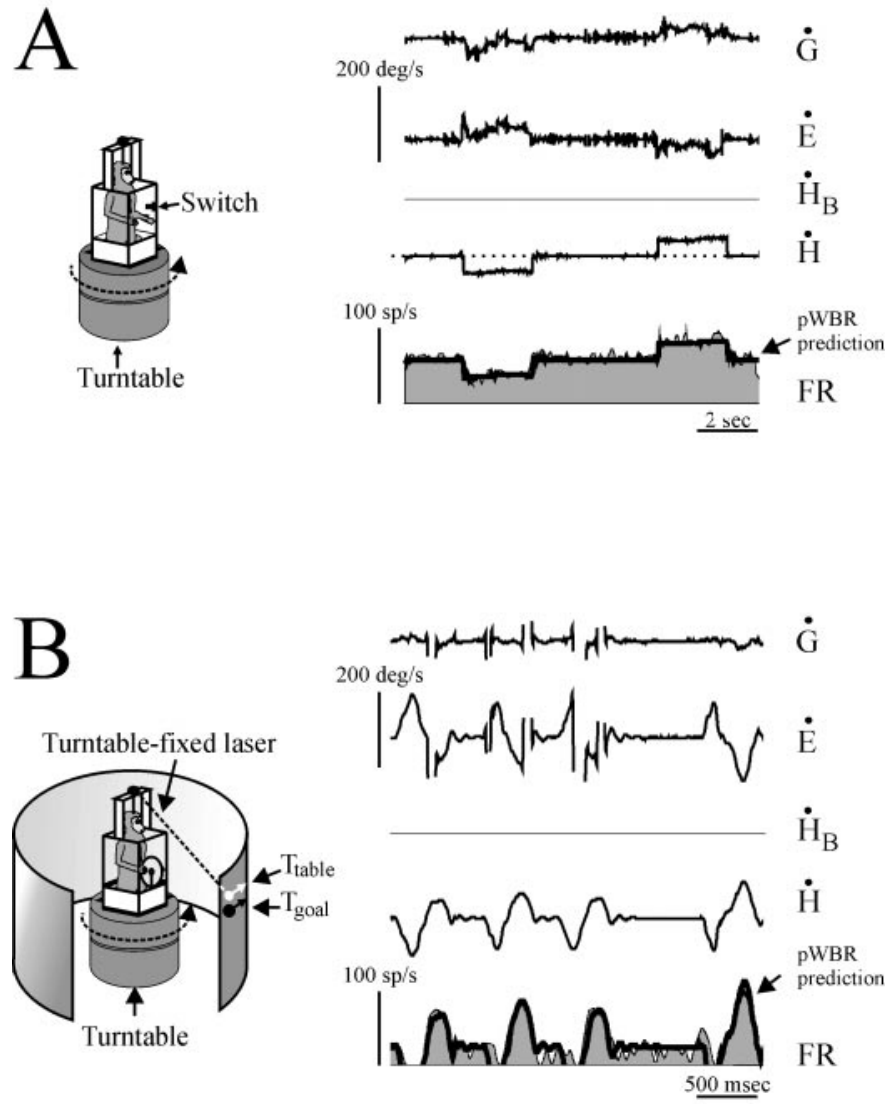

Figure 8. VO neuron responses to voluntary combined head-body motion. $A$, Head-restrained monkeys pressed a switch, which in turn initiated rotation of the turntable (dashed arrow in schema). The activity of an example neuron (unit 103_1) was well predicted by its sensitivity to head velocity during $\mathrm{pWBR}(\mathrm{pW} B R$ prediction, thick trace). A velocity of $0 \% \mathrm{sec}$ is indicated by the horizontal dotted line. $B$, Head-restrained monkeys manually controlled a steering wheel to rotate the vestibular turntable relative to space. Their goal was to align a turntable-fixed laser target $\left(T_{\text {table }}\right)$ with a computer-controlled target $\left(T_{\text {goal }}\right)$. Example neuron (unit 141_1) was typical in that its response was well predicted by its sensitivity to head velocity during pWBR ( $p W B R$ prediction, thick trace). $\dot{H}_{B}$ denotes head-on-body motion $\left(0^{\circ} / \mathrm{sec}\right.$ in $\left.A, B\right)$. All other abbreviations as in Figure 1.

$n=4$; mean, $0.06 \pm 0.02$ (spikes/sec) $/(\% / \mathrm{sec})]$. Moreover, during passive rotation of the head-on-body, the head velocity-related modulation of $\mathrm{V}$-pause neurons was comparable with that observed during passive whole-body rotation $(n=7 ; p>0.4)$.

\section{DISCUSSION}

Previous studies in squirrel monkey have demonstrated that VO neurons differentially encode head motion during passive rotation and the active head movements that are generated during gaze shifts (Boyle et al., 1996; McCrea et al., 1996, 1999). In contrast, a previous investigation of VO neurons in rhesus monkey (Khalsa et al., 1987) had reported that neurons similarly encode head velocity signals during passive whole-body rotation and gaze pursuit, suggesting a species difference. Here we found that neuronal responses in rhesus monkey were attenuated by, on average, $\sim 70 \%$ during gaze shifts, a finding that is consistent with the previous studies in squirrel monkey. Furthermore, we showed that VO neurons demonstrate comparable attenuation during self-generated head-on-body movements made when the premotor saccadic burst generator is not active: immediately after gaze shifts and during gaze pursuit (Fig. 11 $\mathrm{A}$, compare black columns). 
A
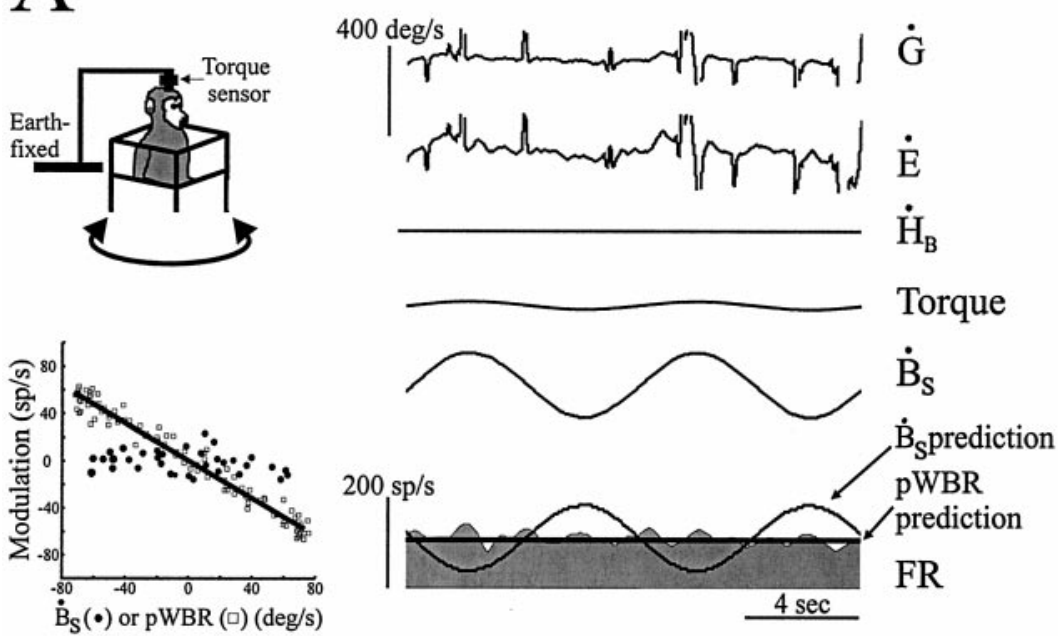

B

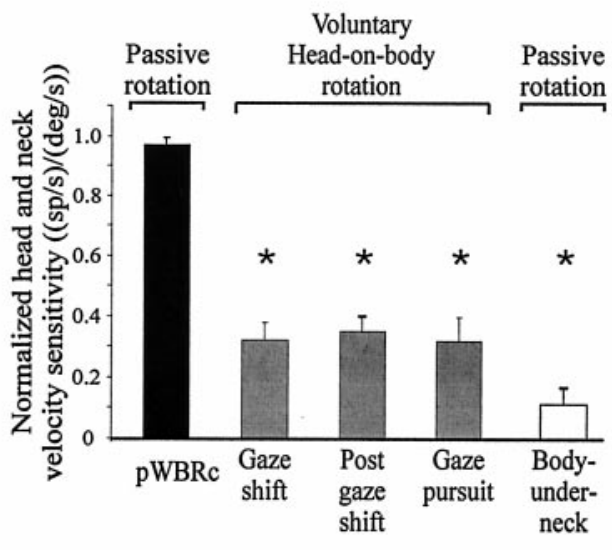

Figure 9. Response of VO neurons to passive neck rotation. $A$, The response of example neuron (unit br31_2) was typical in that it was not modulated when the monkey's body was passively rotated beneath its stationary head; the mean and variance of the discharge of the neuron was comparable during this paradigm and head-restrained eye movement paradigms (mean interspike interval, $18 \pm 13 \mathrm{vs} 17 \pm 13 \mathrm{msec}$, respectively). The neural discharge was well described by the $\dot{\mathrm{H}}_{\mathrm{B}}$ prediction, which was based on head motion (zero in the paradigm), and was overestimated by the $\dot{\mathrm{B}}_{\mathrm{S}}$ prediction, which was generated using the hypothetical neck sensitivity required to account for the attenuated vestibular response of the neuron during active head-on-body motion. Bottom left panel, Comparison of neural modulation in response to pWBR ( $\square$ ) and to passive neck rotation $(\bullet)$. $B$, The normalized neck velocity sensitivity (white column) is insufficient to account for the attenuation observed during gaze shifts, after gaze shifts, and during gaze pursuit (gray columns). Error bars show SEM. Abbreviations as in Figure 1.

A
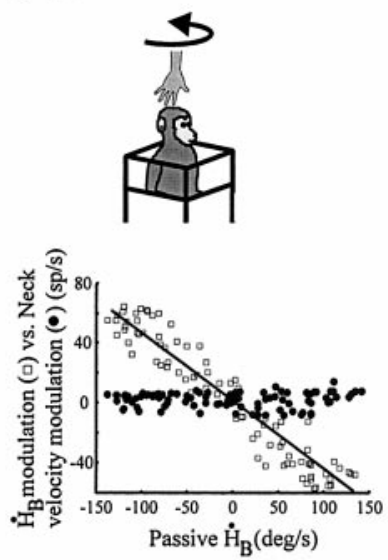

$400 \mathrm{deg} / \mathrm{s}$<smiles>C1CCC2CCC2C1</smiles>

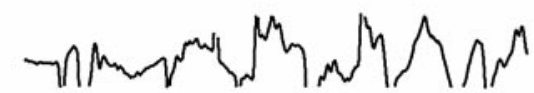

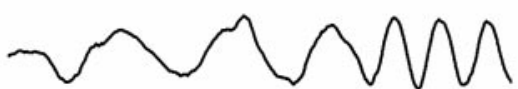

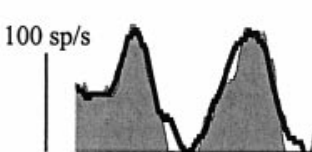

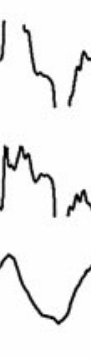
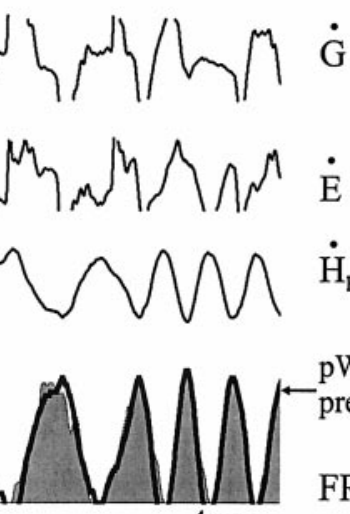

pWBR

prediction

FR
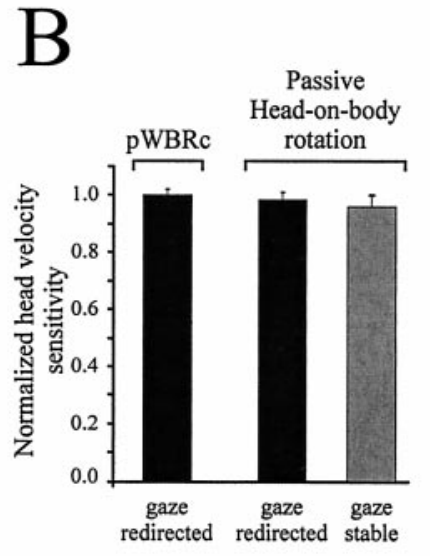

Figure 10. Response of VO neurons to passive rotation of the head-on-body. $A$, The experimenter (hand in schema) passively rotated the monkey's head relative to its earth stationary body. The discharge of example neuron (unit br31_2) was reliably predicted by the sensitivity of the neuron to pWBR (thick trace). Bottom left panel, Relationships between neural modulation $(\square)$ and residual modulation $\left(\mathbf{0}\right.$; total discharge $-\dot{\mathrm{H}}_{\mathrm{B}}$-related modulation) and head-in-space motion (where $\dot{\mathrm{H}}_{\mathrm{S}}=\dot{\mathrm{H}}_{\mathrm{B}}$ ). $B$, For our sample of VO neurons, responses during passive head-on-body rotation were comparable with those resulting passive whole-body rotation [pWBR (used for normalization) and pWBRc (leftmost black column)], regardless of whether the monkey was redirecting (black columns) or stabilizing ( gray column) its gaze. Error bars show SEM. Abbreviations as in Figure 1.

The discrepancy between our study and that of Khalsa et al. (1987) may be the result of two factors: a sampling bias in their data set and/or a difference in the analysis approach. First, it is possible that these investigators inadvertently excluded the VO neurons that showed the greatest attenuation by assuming that they had lost neuronal isolation during the transition from the head-restrained to head-unrestrained condition. Indeed, we were initially concerned that this might have been the case in the present study and were therefore careful to confirm neuronal isolation (see Materials and Methods). Second, these authors used a regression analysis to relate mean firing frequencies to mean head velocities, whereas we used a more sensitive dynamic analysis method to quantify VO neuron discharges. Thus, if their data set included only those VO neurons that showed the least attenuation, it is possible that their analysis was not sufficient to provide evidence that neuronal head velocity sensitivities were altered.

In the present study, we also describe the behavior of a second 


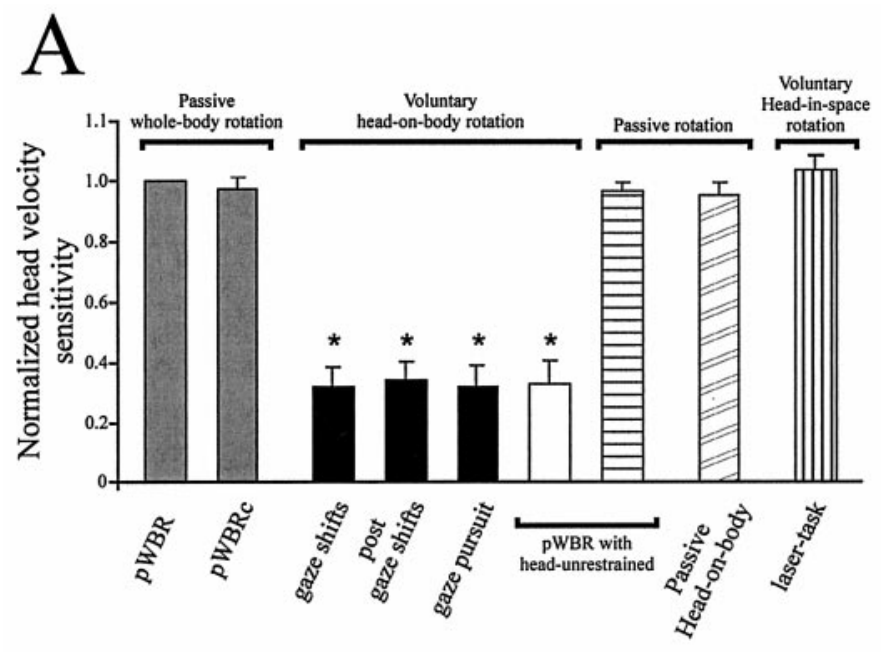

$\mathrm{B}$

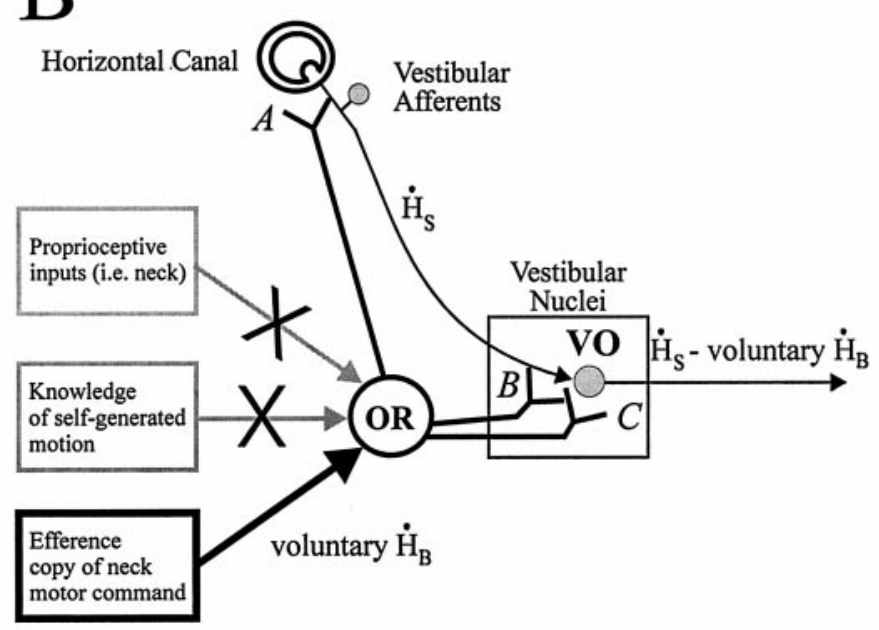

C

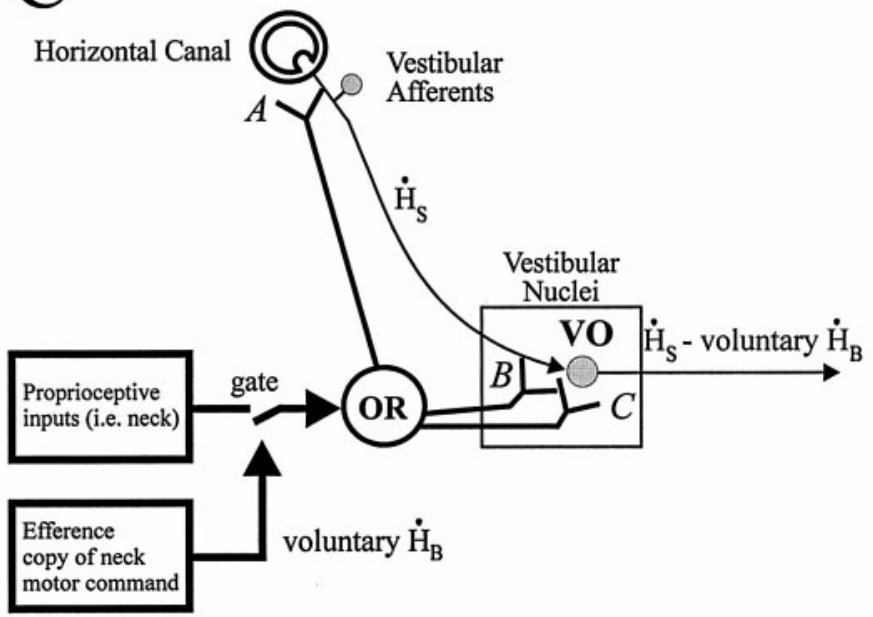

Figure 11. Vestibular reafferent information is differentially processed at the level of the vestibular nuclei. $A$, The responses of VO neurons were significantly suppressed during voluntary head-on-body movements (black columns) compared with pW BR ( gray columns). Similarly, when monkeys made voluntary head-on-body movements during passive rotation, neuronal responses to the voluntary component (open column) remained attenuated, whereas the responses to the passive component (horizontally class of vestibular nuclei neurons, V-pause neurons, that differed from VO neurons in that their sensitivity to head velocity information is reduced to a much greater extent during gaze shifts than during other active motion of the head-on-body. We propose that the preferential suppression of $\mathrm{V}$-pause neuron responses during gaze shifts is mediated via two mechanisms: one similar to that of VO neurons and the other a suppression of activity by the saccadic burst generator (Cullen and Guitton, 1997b). This latter mechanism could be mediated, in part, by projections of premotor burst neurons to type II vestibular neurons (Sasaki and Shimazu, 1981), which in turn send inhibitory projections to neurons within the vestibular nucleus (Shimazu and Precht, 1966). However, this pathway is unlikely to contribute to the attenuation of VO neuron head velocity responses given that they are similarly reduced for all active head-on-body movements.

\section{Negligible role of neck afferent inputs}

Our finding that the activation of neck proprioceptors did not significantly influence the firing patterns of VO neurons was unexpected in light of previous studies (McCrea et al., 1996, 1999; Gdowski and McCrea, 1999). These studies reported that in squirrel monkey most, if not all, secondary vestibular neurons (including VO neurons) are sensitive to passive rotation of the neck. In contrast, we used a comparable experimental approach and concluded that passive activation of neck proprioceptors had little effect on VO responses in rhesus monkey. We found that (1) passive rotation of the monkey's head on its body elicited responses comparable with those elicited by passive whole-body rotation (Fig. 11A, diagonally striped column), and (2) VO neurons were not sensitive to passive rotation of the monkeys body under its stationary head. We propose that the discrepancy between our results and those of McCrea and colleagues might result from either of two factors. First, squirrel monkeys have a relatively small oculomotor range (approximately $\pm 20^{\circ}$ ) (Cullen et al., 1991) compared with humans and rhesus monkeys (approximately $\pm 50^{\circ}$ ) and thus rely more heavily on head motion to redirect gaze. Therefore, it is conceivable that neck proprioception information is processed differently in the two species. Second, because these investigators did not measure neck torque during the paradigms they used to passively activate neck proprioceptors, the possibility that the monkeys generated some resistance (i.e., a non-negligible efference copy signal) cannot be ruled out.

\section{Neck efference copy and the principle of reafference}

Our results are consistent with von Holst and Mittelstaedt's (1950) original idea of reafference, in which an efference copy

\footnotetext{
striped column) remained encoded. In contrast, neurons continued to encode head-in-space motion when the head was passively rotated on the body (diagonally striped column) and when the monkey generated voluntary head-in-space motion by driving the motion of the vestibular turntable (vertically striped column). Error bars show SEM. B, Proposed mechanism for the selective processing of vestibular information by VO neurons during voluntary head-on-body motion. An efferent copy of the neck motor command (voluntary $\dot{H}_{B}$ ) is subtracted from the vestibular afferent-related signal $\left(\dot{\mathrm{H}}_{\mathrm{S}}\right)$ at the level of either the semicircular canals $(A)$, presynaptic to the VO neurons $(B)$, and/or at the VO neuron itself $(C)$. Neither neck proprioceptive information nor knowledge of selfgenerated head-in-space motion contributes to the differential processing of self-generated head-on-body motion. (Note that the hypothetical pathways that have been eliminated are indicated by $X$.) $C$, Alternatively, an efference copy of the neck motor command could function to selectively gate in inhibitory neck proprioceptive inputs.
} 
signal is combined with the vestibular afferent signal to selectively remove the reafferent component caused by the monkey's behavior. We propose that attenuation is mediated by a direct efference copy of the neck motor command input to the VO neurons (Fig. $11 B$ ) or alternatively by efference copy signal that functions to selectively "gate in" inhibitory neck proprioceptive inputs (Fig. $11 C$ ). However, the site of this behaviorally dependent gating of vestibular information is, as yet, unknown. Modulation of sensory information could occur at the level of the semicircular canals themselves (Fig. $11 B$, site $A$ ) via the vestibular efferent system. It is possible that this efferent feedback is used to tune the sensitivity of the vestibular nerve (or a subset of afferents) to voluntary head-on-body movements (Goldberg and Fernandez, 1980). Although this idea is supported by the finding that the toadfish efferent system selectively modulates the activity of afferent fibers during self-motion (i.e., swimming behavior; Highstein, 1992), it has not been tested in primates. Conversely, modulation may occur in the vestibular nucleus itself, either presynaptically (Fig. $11 B$, site $B)$ or at the level of second-order vestibular neurons (Fig. $11 B$, site $C$ ). Additional experiments are needed to determine the location of the behaviorally dependent modulation. Additional experiments will also be required to determine whether vestibular reafference is suppressed during other naturally occurring behaviors. Although neurons responded robustly during the cognitively demanding driving task (Fig. 11 A, vertically striped column), it is possible that, during locomotion and/or combined eye-head-body gaze shifts, the motor efference signals generated by the activation of the head, torso, and limb musculature might collectively influence the response of vestibular nuclei neurons to self-generated head motion in space. Pending the results from these experiments, we suggest that a more suitable name for VO neurons would be vestibular reafference gated (VRG) neurons.

\section{Behaviorally dependent modulation in the vestibular nucleus}

The attenuation of head velocity signals encoded by VO neurons was similar during all active head-on-body movements, regardless of whether the animal was stabilizing its gaze or redirecting its gaze to a new point in space. For example, the head movement sensitivity of VO neurons is attenuated not only during combined eye-head gaze shifts (Fig. 2 A, filled arrows) and pursuit (Fig. 3A) but also immediately after gaze shifts, when gaze is stable in space but the head is still moving (Fig. $2 A$, open arrows). In contrast, the head velocity-related activity of another class of vestibular nuclei neurons, VOR interneurons (i.e., position-vestibularpause neurons that also receive direct inputs from the vestibular nerve but project to the extraocular motor nuclei) is attenuated only while gaze is being redirected in space (Roy and Cullen, 1998), regardless of whether the head motion is actively or passively generated. The difference in behaviorally dependent modulation of these two cell types is consistent with the their role in mediating the vestibulo-collic reflex (see below) and VOR, respectively.

\section{Functional implications}

Emerging evidence suggests that the VCR, a reflex that functions to stabilize the head in space via activation of the neck musculature during head motion, is mediated, at least in part, by VO neurons that project directly to the ventromedial funiculus of segments C1-C2 of the spinal cord (Wilson et al., 1990; Boyle et al., 1996; Gdowski and McCrea, 1999). Because the stabilization response produced by the VCR would be counterproductive during voluntary behaviors in which an animal's goal is to move its head on its body, it would be logical to selectively attenuate VO neuron responses to voluntary head-on-body movements. Accordingly, the VCR would be effectively suppressed for self-generated head motion but would remain responsive to unexpected perturbations of the head. Indeed, we found that, when a monkey actively rotates its head on its body while undergoing passive whole-body rotation, VO neurons continued to encode the passive component $\left(\dot{\mathrm{B}}_{\mathrm{S}}\right)$ of the head-in-space movement (Fig. 11 $\mathrm{A}$, horizontally striped column). This result is in agreement with previous studies (Boyle et al., 1996; McCrea et al., 1999). Furthermore, we discovered for combined active and passive head motion that neuronal responses to the component of head-inspace velocity produced by active head-on-body motion were weak and comparable with those observed during active head-onbody motion in the absence of simultaneous passive rotation (Fig. 11A, compare open columns with black columns).

The information encoded by VO neurons could also be combined with other vestibular pathways to produce an estimate of our current orientation in space during self-generated motion. VO neurons are well situated within extensive cerebellar and cortical recursive networks. The nodulus-uvula of the cerebellum receives inputs from vestibular afferents (Korte and Mugnaini, 1979) and is thought to be reciprocally connected to VO neurons (Wylie et al., 1994; Voogd et al., 1996; Wearne et al., 1998). The transformation of head-centered motion information into an inertial (gravity-centered) coordinate frame requires the nodulusuvula (Angelaki and Hess, 1995; Wearne et al., 1998). In addition, many cortical areas involved in spatial representation, navigation, and gaze control receive vestibular information and in turn project back to the vestibular nuclei (for review, see Fukushima, 1997). For example, posterior parietal neurons have been shown to encode body-referenced and world-referenced information in two separate streams (Snyder et al., 1998) and project via direct and polysynaptic pathways to the vestibular nuclei (FaugierGrimaud and Ventre, 1989). Indeed, it has been suggested recently that VO neurons transform vestibular head-in-space information into body-in-space coordinates (Gdowski and McCrea, 1999). However, although this proposal is consistent with the observation that VO neurons reliably encode passive head-inspace motion during simultaneous passive whole-body rotation and active head-on-body motion, it cannot account for our finding that neurons continue to encode head-in-space velocity during passive rotation of the head on a stationary body. Thus, VO neurons do not simply transform head-in-space signals into bodyin-space coordinates but rather encode vestibular information from which self-generated head-on-body motion has been selectively eliminated. We suggest that this behaviorally dependent processing of vestibular information contributes to both the control and maintenance of posture and the computation of an internal estimate of spatial orientation.

\section{REFERENCES}

Anastasopoulos D, Mergner T (1982) Canal-neck interaction in vestibular nuclear neurons of the cats. Exp Brain Res 46:269-280.

Angelaki DE, Hess BJM (1995) Inertial representation of angular motion in the vestibular system of rhesus monkeys. II. Otolith-controlled transformation that depends on an intact cerebellar nodulus. J Neurophysiol 73:1729-1751.

Bell C (1981) An efference copy which is modified by reafferent input. Science 214:450-453.

Boyle R, Pompeiano O (1981) Responses of vestibulospinal neurons to sinusoidal rotation of the neck. J Neurophysiol 44:633-649.

Boyle R, Belton T, McCrea RA (1996) Responses of identified vestibu- 
lospinal neurons to voluntary eye and head movements in the squirrel monkey. Ann NY Acad Sci 781:244-263.

Cullen KE, Guitton D (1997a) Analysis of primate IBN spike trains using system identification techniques. I. Relationship to eye movement dynamics during head-fixed saccades. J Neurophysiol 78:3259-3282.

Cullen KE, Guitton D (1997b) Analysis of primate IBN spike trains using system identification techniques. II. Relationship to gaze, eye, and head movement dynamics during head-free gaze shifts. J Neurophysiol 78:3283-3306.

Cullen KE, McCrea RA (1993) Firing behavior of brain stem neurons during voluntary cancellation of the horizontal vestibulo-ocular reflex. I. Secondary vestibular neurons. J Neurophysiol 70:828-843.

Cullen KE, Belton T, McCrea RA (1991) A non-visual mechanism for voluntary cancellation of the vestibulo-ocular reflex. Exp Brain Res 83:237-252.

Cullen KE, Chen-Huang C, McCrea RA (1993a) Firing behavior of brain stem neurons during voluntary cancellation of the horizontal vestibulo-ocular reflex. II. Eye-movement related neurons. J Neurophysiol 70:844-856.

Cullen KE, Guitton D, Rey CG, Jiang W (1993b) Gaze-related activity of putative inhibitory burst neurons in the head-free cat. J Neurophysiol 70:2678-2683.

Cullen KE, Rey CG, Guitton D, Galiana HL (1996) The use of system identification techniques in the analysis of oculomotor burst neuron spike train dynamics. J Comput Neurosci 3:347-368.

Edwards DH, Heitler WJ, Krasne FB (1999) Fifty years of a command neuron: the neurobiology of escape behavior in the crayfish. Trends Neurosci 22:153-161.

Faugier-Grimaud S, Ventre J (1989) Anatomic connections of inferior parietal cortex (area 7) with subcortical structures related to vestibuloocular function in a monkey (Macaca fascicularis). J Comp Neurol 280:1-14.

Fuchs AF, Kimm J (1975) Unit activity in vestibular nucleus of the alert monkey during horizontal angular acceleration and eye movement. J Neurophysiol 38:1140-1161.

Fuchs AF, Robinson DA (1966) A method for measuring horizontal and vertical eye movements in the monkey. J Physiol (Lond) 191:609-631.

Fukushima K (1997) Corticovestibular interactions: anatomy, electrophysiology, and functional considerations. Exp Brain Res 117:1-16.

Fuller JH (1988) Chronic recording of neck sensory input to vestibular neurons. In: Control of head movement (Peterson BW, Richmond FJ, eds), pp120-128. Oxford: Oxford UP.

Gdowski GT, McCrea RA (1999) Integration of vestibular and head movement signals in the vestibular nuclei during whole-body rotation. J Neurophysiol 81:436-449.

Goldberg JM, Fernandez C (1980) Efferent vestibular system in the squirrel monkey: anatomical location and influence on afferent activity. J Neurophysiol 43:986-1025.

Hays AV, Richmond BJ, Optican LM (1982) A UNIX-based multiple process system for real-time data acquisition and control. W ESCOM Conf Proc 2:1-10.

Highstein SM (1992) The efferent control of the organs of balance and equilibrium in the toadfish, Opsanus tau. Ann NY Acad Sci 656:108-123.

Hulliger M, Matthews PBC, Noth J (1977) Static and dynamic fusimotor action on the response of IA fibers to low frequency sinusoidal stretching of widely ranging amplitude. J Physiol (Lond) 267:811-838.

Keller EL (1974) Participation of medial pontine reticular formation in eye movement generation in monkey. J Neurophysiol 37:316-332.

Keller EL, Kamath BY (1975) Characteristics of head rotation and eye movement-related neurons in alert monkey vestibular nucleus. Brain Res 100:182-187.

Khalsa SB, Tomlinson RD, Schwarz DW, Landolt JP (1987) Vestibular nuclear neuron activity during active and passive head movement in the alert rhesus monkey. J Neurophysiol 57:1484-1497.
Khalsa SB, Tomlinson RD, Scharwz DW (1988) Secondary vestibular and neck position signals in the vestibular nuclei of alert rhesus monkeys performing active head movements. Acta Otolaryngol 106:269-275.

Korte G, Mugnaini E (1979) The cerebellar projection of the vestibular nerve in the cat. J Comp Neurol 184:265-278.

Krasne FB, Bryan JS (1973) Habituation: regulation through presynaptic inhibition. Science 182:590-592.

McCrea RA, Chen-Huang C, Belton T, Gdowski GT (1996) Behavior contingent processing of vestibular sensory signals in the vestibular nuclei. Ann NY Acad Sci 781:292-303.

McCrea RA, Gdowski GT, Boyle R, Belton T (1999) Firing behavior of vestibular neurons during active and passive head movements: vestibulo-spinal and other non-eye-movement related neurons. J Neurophysiol 82:416-428.

Prochazka A, Hulliger M, Trend P, Durmuller N (1987) Dynamic and static fusimotor set in various behavioural contexts. In: Mechanoreceptors. Development, structure, and function (Hnik P, Soukup T, Vejsada R, Zelena J, eds), pp 417-430. New York: Plenum.

Roy JE, Cullen KE (1998) A neural correlate for vestibulo-ocular reflex suppression during voluntary eye-head gaze shifts. Nat Neurosci $1: 404-410$.

Roy JE, Cullen KE (1999) Differential processing of head velocity signals in the vestibular nuclei: voluntary head/neck versus head/space motion. Soc Neurosci Abstr 25:265.

Sasaki S, Shimazu H (1981) Reticulovestibular organization participating in generation of horizontal fast eye movement. Ann NY Acad Sci 374:130-145.

Sato H, Ohkawa T, Uchino Y, Wilson VJ (1997) Excitatory connections between neurons of the central cervical nucleus and vestibular neurons in the cat. Exp Brain Res 115:381-386.

Scudder CA, Fuchs AF (1992) Physiological and behavioural identification of vestibular nucleus neurons mediating the horizontal vestibuloocular reflex in trained rhesus monkeys. J Neurophysiol 68:244-264.

Shimazu H, Precht W (1966) Inhibition of central vestibular neurons from the contralateral labyrinth and its mediating pathway. J Neurophysiol 29:467-492.

Snyder LH, Grieve KL, Brotchie P, Andersen RA (1998) Separate body- and world-referenced representations of visual space in parietal cortex. Nature 394:887-891.

Sylvestre PA, Cullen KE (1999) Quantitative analysis of abducens neuron discharge dynamics during saccadic and slow eye movements. J Neurophysiol 82:2616-2632.

Tomlinson RD, Robinson DA (1984) Signals in vestibular nucleus mediating vertical eye movements in the monkey. J Neurophysiol $51: 1121-1136$.

Voogd J, Gerrits M, Ruigrok JH (1996) Organization of the vestibulocerebellum. Ann NY Acad Sci 781:553-579.

von Holst E, Mittelstaedt H (1950) Das reafferenzprinzip. Naturwissenschaften 37:464-476.

Wearne S, Raphan T, Cohen B (1998) Control of spatial orientation of the angular vestibuloocular reflex by the nodulus and uvula. J Neurophysiol 79:2690-2715.

Wilson VJ (1991) Vestibulospinal and neck reflexes: interaction in the vestibular nuclei. Ach Ital Biol 129:43-52.

Wilson VJ, Yamagata Y, Yates BJ, Schor RH, Nonaka S (1990) Response of vestibular neurons to head rotations in vertical planes. III. Response of vestibulocollic neurons to vestibular and neck stimulation. J Neurophysiol 64:1695-1703.

Wylie DR, De Zeeuw CI, Digiorgi PL, Simpson JI (1994) Projections of individual purkinje cells of identified zones in the ventral nodulus to the vestibular and cerebellar nuclei in the rabbit. J Comp Neurol 349:448463. 